\title{
COMPARAÇÃO DA QUALIDADE DE SELAMENTO PERIFÉRICO DE DIFERENTES MATERIAIS RESTAURADORES PROVISÓRIOS
}

\section{COMPARISON OF SEALING ABILITY OF DIFFERENT TEMPORARY RESTORATIVE MATERIALS}

\author{
Paloma Mariana Ramos Bitencourt \\ Maria Leticia Borges Britto* \\ Cleber Keiti Nabeshima**
}

\begin{abstract}
RESUMO
Diferentes materiais restauradores temporários podem ser utilizados durante tratamento endodôntico, porém é necessário possuir excelentes propriedades de selamento periférico. Assim, o objetivo deste estudo é comparar a infiltração marginal em diferentes materiais restauradores temporários. Cinquenta e dois molares superiores foram utilizados, onde cinquenta dentes foram abertos até a câmara pulpar, e divididos aleatoriamente: Grupo 1 foi restaurado utilizando-se Bioplic; Grupo 2 foi restaurado utilizando-se Cimpat rosa; Grupo 3 foi restaurado usando-se óxido de zinco e eugenol; Grupo 4 usando-se guta-percha, dois dentes hígidos formaram o grupocontrole negativo e dois dentes acessados e sem restauração foi o grupo controle-positivo. A raiz e o ápice dos dentes foram selados com esmalte, e todas as amostras imersas em azul de metileno a $2 \%$ e mantidos a $37^{\circ}$ por $72 \mathrm{~h}$. Assim, os dentes foram seccionados longitudinalmente e a infiltração linear medida em mm, sendo posteriormente aplicada análise estatística com teste de Kuskall Wallis e teste de Dunn. A maior infiltração ocorreu nos grupos 3 e 4, e as menores ocorreram no grupo 1 e 2 com diferença significante entre os grupos. Pode-se concluir que o Cimpat rosa e o Bioplic são materiais favoráveis no selamento coronário durante tratamento endodôntico.
\end{abstract}

DESCRITORES: Restauração Dentária Temporária • Endodontia • Infiltração Dentária.

\section{ABSTRACT}

Different temporary materials can be used to dental restoration during endodontic treatment, but it is necessary an excellent sealing property, so the aim of this study is compare the leakage of temporary restorative materials. Fifty two third molars were used, which fifty teeth were opened through the pulp chamber, and randomly divided: group 1 was restored using Bioplic; group 2 was restored using Pink Cimpat; group 3 was restored using Zinc oxid eugenol; group 4 was restored using gutta-percha; two not opened teeth were negative control group, and two opened teeth no restored were positive control group. Then it was made completely impermeable of the root and apex of the teeth by varnish. All samples were immersed in $2 \%$ metylene blue and kept at $37^{\circ} \mathrm{C}$ for $72 \mathrm{~h}$. After longitudinal sectioning the leakage was measured in $\mathrm{mm}$ and statistical analysis was performed using Kruskall Wallis and Dunn test. It was significant difference between groups, which greater linear leakeage on the groups 3 and 4, and less linear leakage on the groups 1 and 2. It can conclude that pink Cimpat and Bioplic are temporary restorative materials satisfactory on sealing ability during endodontic treatment.

DESCRIPTORS: Dental Restoration Temporary • Endodontics • Dental Leakage.

\footnotetext{
* Especializanda em Endodontia pela Universidade Cruzeiro do Sul (UNICSUL).

** Professora Coordenadora do Curso de Especialização em Endodontia da Universidade Cruzeiro do Sul (UNICSUL). Doutora em Endodontia pela Faculdade de Odontologia da Universidade de São Paulo (FOUSP).

*** Mestrando em Endodontia pela Faculdade de Odontologia da Universidade de São Paulo (FOUSP). Professor do Curso de Especialização em Endodontia da Universidade Cruzeiro do Sul (UNICSUL).
} 
COMPARAÇÃO

DA QUALIDADE

DE SELAMENTO

PERIFÉRICO

DE DIFERENTES

MATERIAIS

RESTAURADORES

PROVISORIOS

\section{4}

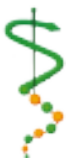

REVISTA DE

ODONTOLOGIA DA

UNI VERS I DADE

CIDADE DE SÃO

PAULO

$2010 ; 22(3):$ 223-28, SET-DEZ

\section{IN T RO DUÇ ÃO}

Uma das principais propostas do tratamento endodôntico é atingir a máxima desinfecção do sistema de canais radiculares, no qual deve ser mantida até a restauração definitiva do elemento dentário (Haapasalo et al. ${ }^{1}$ 2003, Salazar-Silva Jr. et al. ${ }^{2}$ 2004).

Para tal, é imprescindível a proteção do acesso coronário, impedindo-se que a limpeza obtida seja perdida pela entrada de fluidos e bactérias provenientes principalmente do meio bucal (Salazar-Silva Jr. et al. ${ }^{2}$ 2004). Acredita-se que maior parte dos insucessos pode estar relacionada ao mau selamento coronário (Haapasalo et al. ${ }^{1}$ 2003).

Diversos materiais têm sido utilizados para essa proteção entre as sessões, diferenciando-se pela sua base e características.

O mais utilizado é o óxido de zinco e eugenol, o qual deve ser preparado pela mistura do pó de óxido de zinco com líquido de eugenol. Essa mistura resulta numa pasta de consistência variada que depende da proporção de pó líquido utilizado. Estudos têm relatado que esse cimento possui maior resistência à compressão em relação a todos os demais (Mota ${ }^{3}$ 2007), no entanto, sua capacidade de vedamento é baixa (Fachin et al. ${ }^{4}$ 2006). Uma comparação realizada entre o IRM, Cimpat branco, e a sua associação, mostrou que o IRM promove infiltração em toda sua extensão de junção com o dente, e nas amostras onde se utilizou a associação ocorreu infiltração somente nas regiões de contato com o IRM, mostrando a má qualidade de selamento do óxido de zinco e eugenol propriamente dito (Palo et al. $\left.{ }^{5}, 1996\right)$.

Devido à praticidade, surgiram os cimentos provisórios à base de óxido de zinco pronto, que dispensa a incorporação pó líquido, havendo presa do material após a sua inserção e contato com a saliva. No entanto, esses cimentos possuem queda de resistência à compressão, por isso foi incorporado mais óxido de zinco e de ferro com a finalidade de aumentar a resistência, entre eles o Cimpat rosa, sobre o qual estudos clínicos têm apresentado resultados altamente satisfatórios porque é capaz de se manter estável em cavidades de diversos tipos, sem o mínimo de paredes para retenção (Nabeshima e Britto ${ }^{6}$, 2008).

Mais uma opção para a restauração provisória foram os cimentos provisórios à base de resina; pelo fato de serem incolores são mais estéticos, principalmente em dentes anteriores, numa fase intermediária de tratamento; além disso são compatíveis com resinas compostas que não polimerizam na presença de eugenol. A avaliação da microinfiltração de dois cimentos restauradores provisórios fotopolimerizáveis mostrou excelente capacidade de selamento periférico (Bitencourt et al. ${ }^{2} 2010$ ).

Outro material que já faz parte do arsenal endodôntico e poderia ser utilizado é a guta percha, que após sua plastificação é levada à cavidade de forma simples e rápida.

Visto que as diferentes formulações e bases podem influenciar diretamente na capacidade de selamento, o objetivo deste trabalho é comparar o vedamento periférico proporcionado por diferentes tipos de materiais restauradores provisórios utilizados em Endodontia.

\section{MATERIAL E MÉTODOS}

O presente estudo foi submetido e aprovado pelo comitê de ética em pesquisa da instituição de acordo com a Declaração de Helsink (2000).

Para este estudo foram utilizados 52 terceiros molares humanos hígidos, cujas raízes e ápices foram impermeabilizados com esmalte incolor para unhas, nos quais 50 amostras tiveram acesso à câmara pulpar perfazendo uma classe I.

Dois dentes sem cirurgia de acesso serviram como controle negativo e dois dentes acessados sem a utilização de restauração provisória foram os controles positivos.

Os demais 48 dentes foram divididos aleatoriamente em 4 grupos, de acordo com o material restaurador provisório utilizado.

G1 - Bioplic (Biodinâmica Ltda, Paraná, Brasil);

G2 - Cimpat Rosa (Septodont, Saint Maur Des Fosses, France); 
G3 - Óxido de Zinco e Eugenol (IRM, Dentsply, Perópolis, Brasil);

G4 - Guta Percha (Dentsply, Petrópolis, Brasil).

Todos os dentes foram mergulhados em azul de metileno a 2\% (O Graal -Farmácia de manipulação, São Paulo, Brasil) e mantidos em estufa (Quimis, Diadema, Brasil) à temperatura de $37^{\circ} \mathrm{C}$ por 72 horas, e lavados em água corrente.

Os dentes foram incluídos em gesso comum, e as amostras foram cortadas longitudinalmente ao longo do eixo do dente no sentido vestíbulo lingual, através de um recortador de gesso, até a região central do dente.

Com auxílio de uma lupa de aumento e régua, a infiltração do corante foi medida em milímetros a partir do ângulo cavo-superficial, na interface material de selamento provisório-esmalte dental, por meio de lupa de aumento.

Os dados obtidos foram submetidos à curva de normalidade que levou ao teste estatístico de Kruskal-Wallis, com nível de significância de 5\%, e a comparação dos grupos foi feita através do teste de Dunn.

\section{RESULTADOS}

O G1 apresentou infiltração linear que variou de 0 a $3 \mathrm{~mm}$, e o G2 variou de 1 a $3 \mathrm{~mm}$; nenhuma amostra de ambos os grupos mostrou infiltração de $5 \mathrm{~mm}$.

No entanto, os grupos 3 e 4 apresentaram infiltração total em todas as amostras do grupo, resultados semelhantes ao obtido pelo grupo-controle positivo.

A análise estatística apresentou diferença significante entre os materiais utilizados $(p<0.0001)$, onde G1 e G2 se apresentaram diferentes de G3 e G4.

Os dados estatísticos podem ser vistos na Tabela 1 e a comparação percentual da quantidade de infiltração linear entre os materiais utilizados pode ser vista no Gráfico 1.

\section{I SCUSSÃO}

O sucesso do tratamento endodôntico não depende somente e exclusivamente do preparo químico cirúrgico, mas também do selamento coronário entre as sessões ou pré-restauração definitiva.
Esse é um momento crítico, no qual poderão ser colocados em risco todos os cuidados tomados durante o procedimento clínico, além disso, a penetração de bactérias mais virulentas ao endodonto torna a terapia mais dificultada.

Espera-se que o material eleito para a restauração provisória tenha algumas características como biocompatibilidade, estabilidade dimensional, resistência à abrasão e compressão e principalmente excelente capacidade de selamento periférico (Balto ${ }^{8}$ 2002).

Devido à disponibilidade de diversos materiais cuja finalidade é a restauração provisória, selecionou-se para este estudo os mais comuns, e com variáveis importantes; por exemplo, o óxido de zinco e eugenol (IRM) propriamente dito é o mais utilizado, a forma pronta à base de óxido de zinco reforçado possui maior praticidade, os resinosos são mais estéticos, e a guta percha faz parte do arsenal endodôntico.

Terceiros molares foram usados pela facilidade de obtenção em relação a outros dentes e por se apresentarem frequentemente hígidos, permitindo uma padronização da abertura coronária por meio da confecção de uma Classe I. O selamento radicular e apical foi feito, pois túbulos dentinários, forames ou defeitos no cemento poderiam influenciar nos resultados, além disso houve padronização de $5 \mathrm{~mm}$ da camada de material restaurador, pois espessura menor que $3 \mathrm{~mm}$ é uma variável que poderia influenciar nos resultados (Weston et al. ${ }^{9}$ 2008).

Os resultados obtidos firmam o encontrado por Fachin et al. ${ }^{4}$ (2007), em que o óxido de zinco e eugenol e a guta percha apresentaram os piores resultados; no presente estudo promoveram infiltração total similar ao observado no grupo-controle positivo. No caso do óxido de zinco e eugenol, este fato pode ser explicado devido à mudança de volume do material pós-presa, pois o mesmo sofre contração durante a reação de presa que resulta em menores dimensões e consequentemente perde sua adaptação marginal. Já a gutapercha possivelmente resultou em infiltração exacerbada devido à falta de adesão, pois não há nenhuma adesividade à pare-
BITENCOURT PMR BRITTO MLB NABESHIMA CK COMPARAÇÃo DA QUALIDADE DE SELAMENTO PERIFÉRICO DE DIFERENTES MATERIAIS RESTAURADORES PROVISÓRIOS
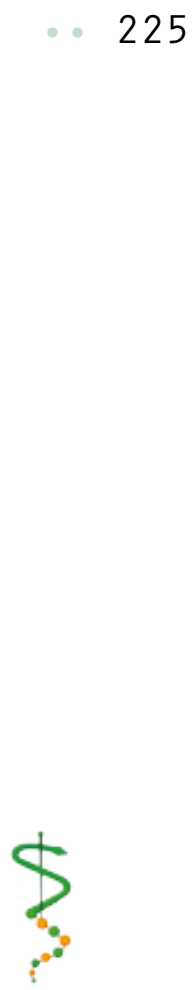

REVISTA DE ODONTOLOGIA DA UN I VERS I DADE Cidade de São PAULO

2010; 22(3): 223-28, SET-DEZ 
BITENCOURT PMR

BRITTO MLB

NABESHIMA CK

COMPARAÇÃO

DA QUALIDADE

DE SELAMENTO

PERIFÉRICO

DE DIFERENTES

MATERIAIS

RESTAURADORES

PROVISORIOS

\section{6}

I SSN 1983-5183

de dentinária.

Os resultados superiores do Cimpat rosa em relação ao óxido de zinco confirmam estudos prévios (Carvalho et al. ${ }^{10}$ 2004, Macedo et al. ${ }^{11}$ 2009), no qual mostram que a adição de óxido de zinco em sua formulação original pode não influenciar na qualidade de selamento do material. Segundo $\operatorname{Mota}^{3}$ (2007), o óxido de zinco e eugenol propriamente dito sofre contração durante sua presa, e o Cimpat rosa sofre expansão devido sua capacidade de absorver água, o que poderia explicar os resultados obtidos. Além disso, a presença de eugenol no óxido de zinco e eugenol limita seu uso na indicação de resina composta, pois impede a polimerização da mesma, fato este que não ocorre com o Cimpat.

Resultados semelhantes ao Cimpat rosa foram obtidos pelo Bioplic e possivelmente se devem à mesma característica, pois segundo Bitencourt et al. ${ }^{7}$ (2010) a absorção de água da saliva leva a uma leve expansão, realizando uma pressão negativa na parede da cavidade, garantindo uma eficiente capacidade seladora, e a contração de polimerização resinosa é mínima, muito menor do que as resinas compostas convencionais, não influenciando no vedamento. Outros autores já mostraram resultados semelhantes entre o Bioplic e outros cimentos provisórios prontos para uso à base de óxido de zinco (Bordin et al. ${ }^{12}$ 2007, Marques et al. ${ }^{13}$ 2005, Seixas et al. ${ }^{14}$ 2008) ou superioridade quando comparado ao óxido de zinco e eugenol (Malmegin et al. ${ }^{15}$ 2008).

Assim, baseado nos resultados obtidos neste trabalho, pode-se concluir que é viável o uso tanto do Bioplic como do Cimpat rosa para o selamento coronário entre as sessões de endodontia, onde o Bioplic parece ser mais indicado em dentes anteriores, devido a sua cor, além de proporcionar mais estética; no entanto, vale lembrar que será necessário o uso de fotopolimerizador que não é um equipamento comum da prática endodôntica.

Além disso, a ausência de trabalhos que avaliam a resistência à compressão de alguns deles incentiva a continuação de estudos, uma vez que resistência é outro ponto de extrema importância para o material restaurador provisório.

\section{CONCLUSÃO}

Diante dos resultados pode-se concluir que o Cimpat rosa e o Bioplic apresentam resultados satisfatórios como restauradores provisórios coronários em Endodontia.

Gráfico 1 - Comparação percentual da infiltração linear entre os materiais testados
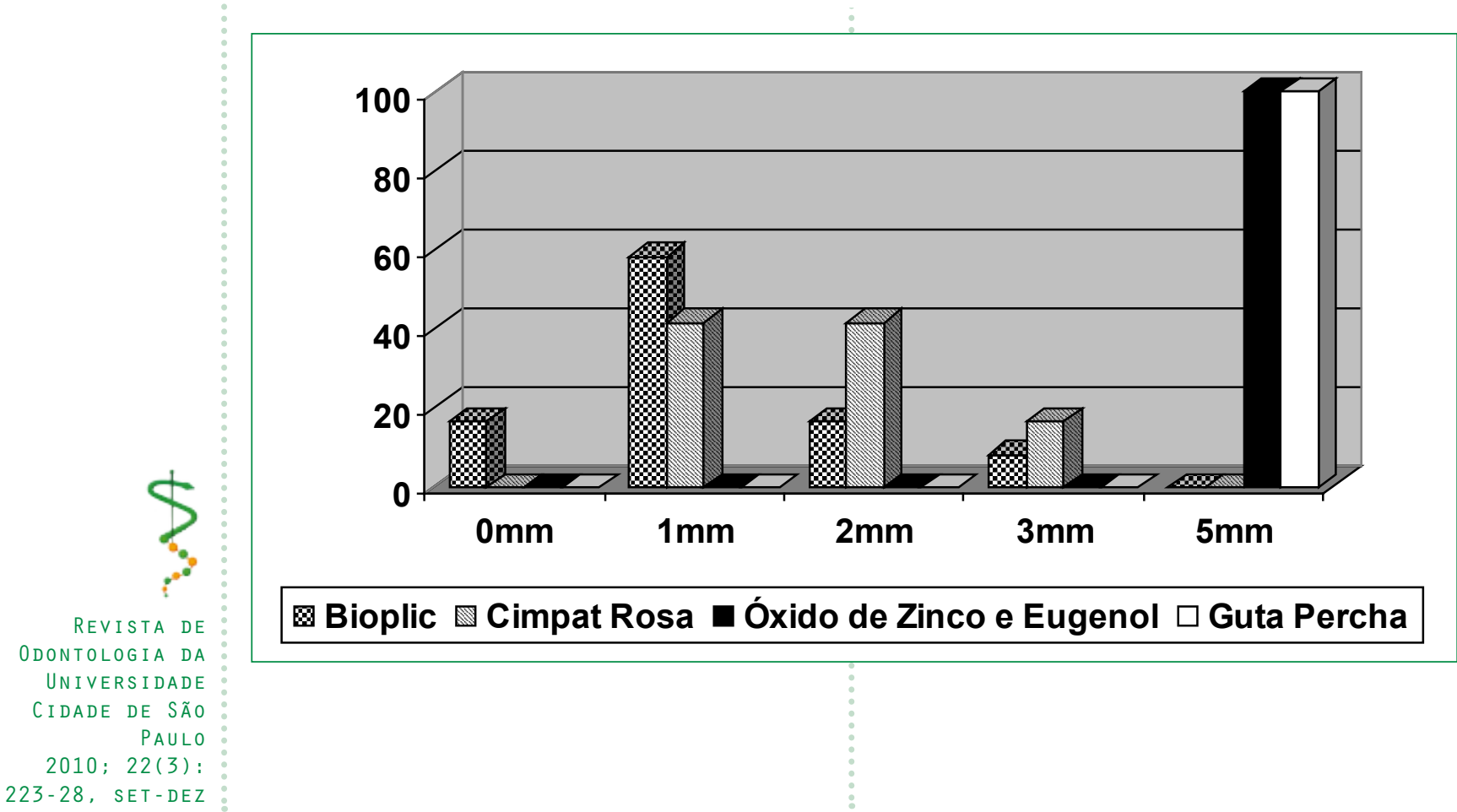
Tabela 1 - Dados Estatísticos da Infiltração linear em milímetros do corante azul de metileno

\begin{tabular}{lllllll}
\hline \hline Grupo & $\mathrm{n}$ & Média & Mediana & Desvio Padrão & Mínimo & Máximo \\
\hline Bioplic $^{\mathrm{a}}$ & 12 & 1.16 & 1.0 & 0.8348 & 0.0 & 3.0 \\
Cimpat Rosa $^{\mathrm{a}}$ & 12 & 1.75 & 2.0 & 0.7538 & 1.0 & 3.0 \\
Óxido de Zinco e Eugenol $^{\mathrm{b}}$ & 12 & 5.0 & 5.0 & 0.0000 & 5.0 & 5.0 \\
Guta Percha $^{\mathrm{b}}$ & 12 & 5.0 & 5.0 & 0.0000 & 5.0 & 5.0 \\
\hline \hline
\end{tabular}

* aXb - Diferença significante $(\mathrm{p}=0.0001)$

\section{REFERÊNCIAS}

1. Haapasalo M, Udnæs T, Endal U. Persistent, recurrent, and acquired infection of the root canal system post-treatment. Endod Topics 2003 Nov; 6(1):29-56.

2. Salazar-Silva JR, Pereira RCS, Ramalho LMP. Importância do selamento provisório no sucesso do tratamento endodôntico. Pesq Bras Odontoped Clinic Integr 2004 mai-ago; 4(2): 143-9.

3. Mota KS. Comparação in vitro da resistência de diferentes cimentos restauradores provisórios. [Trabalho de Conclusão de Curso de Graduação]. São Paulo: Universidade Cruzeiro do Sul; 2007.

4. Fachin EVF, Perondi M, Grecca FS. Comparação da capacidade de selamento de diferentes materiais restauradores provisórios. RPG Rev Pos-grad. 2006 out-dez; 13(4): 292-8.

5. Polo I, Lage Marques JLS, Cardoso RJA, et al. Selamento marginal simples e duplo em endodontia. Rev Assoc Paul Cirur Dent 1996 set-out; 50(5): 435-39.

6. Nabeshima CK, Britto MLB. Avaliação da resistência do Cimpat $®$ rosa como restauração temporária durante tratamento endodôntico. Int J Dent. 2008; 7(3): 158-61.

7. Bitencourt PMR, Britto MLB, Nabeshima CK. Avaliação do selamento provisório de dois cimentos provisórios fotopolimerizáveis utilizados em Endodontia. RSBO 2010; 7(3): 269-74.

8. Balto $\mathrm{H}$. An assement of microbial coronal leakage of temporary filling materials in endodontically. J Endod 2002 Nov; 28(11): 762-4.

9. Weston $\mathrm{CH}$, Barfield RD, Ruby JD, Litaker MS, McNeal SF, Eleazer PD. Comparison of preparation design and material thickness on microbial leakage through Cavit using a tooth model system. Oral Surg Oral Med Oral Pathol Oral Radiol Endod. 2008 Apr; 105(4): 530-5.

10. Carvalho GL, Rola PP, Cruz CW, et al. Avaliação da infiltração marginal em dois cimentos provisórios em dentes com e sem preparo do bisel do ângulo cavo superficial. Cienc Odontol Bras 2004 abr-jun; 7(2): 41-6.

11. Macedo RG, Nabeshima CK, Britto MLB. Microinfiltração do óxido de zinco e eugenol e do cimpat rosa como restaurador provisório. Arq Bras Odontol 2009; 5(2): 49-52.

12. Bordin MM, Coradini PC, Salles AA, et al. Avaliação, in vitro, da microinfiltração coronária na interface amálgama de prata e três materiais restauradores provisórios de uso em Endodontia. Rev Fac Odontol Porto Alegre. 2007; 48(1/3): 82-7.

13. Marques MOA, Paiva TPF, Soares S, et al. Avaliação da infiltração marginal em materiais restauradores temporários - Um estudo in vitro. Pesq Bras Odontoped Clin Integr. 2005 jan-abr; 5(1): 47-52.

BITENCOURT PMR BRITTO MLB NABESHIMA CK COMPARAÇÃO DA QUALIDADE DE SELAMENTO PERIFÉRICO DE DIFERENTES MATERIAIS RESTAURADORES PROVISORIOS

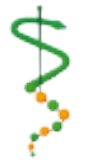

REVISTA DE ODONTOLOGIA DA UNI VERS I DADE Cidade de são PAULO 2010; 22(3): 223-28, SET-DEZ 
BITENCOURT PMR

BRITTO MLB NABESHIMA CK

COMPARAÇÃO DA QUALIDADE DE SELAMENTO PERIFÉRICO DE DIFERENTES MATERIAIS RESTAURADORES PROVISORIOS
14. Seixas FH, Martinelli DF, Cecchin D, Ribeiro RG, Silva RS, Pécora JD. Avaliação ex vivo da microinfiltração marginal coronária de resauradores provisórios usados em endodontia. RFO UFP. 2008 set-dez; 13(3): 31-5.

15. Malmegrin LA, Oliveira C, Cervi DA, Almeida MJP, Zuza EP, Aielo OE, Pereira LA. Estudo comparativo da capacidade seladora de um fotopolimerizável temporário. Rev Assoc Paul Cir Dent. 2008 jan-fev; 62(1): 44-8.

Recebido em: 03/08/2010

Aceito em: 18/09/2010 